\title{
Diffusion of electrons in random magnetic fields
}

\author{
Tohru Kawarabayashi and Tomi Ohtsuki* \\ Institute for Solid State Physics, University of Tokyo, \\ Roppongi, Minato-ku, Tokyo 106, Japan \\ ${ }^{*}$ Department of Physics, Faculty of Science, Toho University, Miyama 2-2-1, \\ Funabashi 274, Japan
}

\begin{abstract}
Diffusion of electrons in a two-dimensional system in static random magnetic fields is studied by solving the time-dependent Schrödinger equation numerically. The asymptotic behaviors of the second moment of the wave packets and the temporal auto-correlation function in such systems are investigated. It is shown that, in the region away from the band edge, the growth of the second moment of the wave packets turns out to be diffusive, whereas the exponents for the power-law decay of the temporal auto-correlation function suggest a kind of fractal structure in the energy spectrum and in the wave functions. The present results are consistent with the interpretation that the states away from the band edge region are critical.
\end{abstract}

PACS numbers: 05.70.Fh(Phase Transitions : general aspect), 71.30 (MetalInsulator transitions), 71.55.J(Localization in disordered structures),73.20(Electronic surface states) 


\section{$\S 1$. Introduction}

Since the development of the scaling theory of localization [1, 2], much work has been done on the transport properties of disordered electron systems [3, [4]. To understand the transport properties of a random system, the concept of quantum interference plays an important role. In fact, in the last decade, many interesting and universal phenomena observed in mesoscopic systems have been understood in terms of the quantum interference [5, 6]. In most cases, however, only a coherent scattering due to the random potentials has been discussed. Recently there has been a growing interest on the coherent scattering by the randomly varying magnetic fields [7] 10$]$. In particular, considerable attention has been paid to transport properties in twodimensional systems in randomly distributed magnetic fluxes. For instance, several authors [11-19] have discussed whether or not the Anderson transition occurs in a two-dimensional system in static random magnetic fields. A possibility of the An-

derson transition in such a system has been proposed in references [13], [15] and [19] based on numerical calculations of conductance and the scaling analysis for the exponential-decay length of the Green function in quasi-one-dimensional systems. According to the references [13] and [15], the mobility edge $E_{c}$ has been estimated as $E_{c}=2.6 \sim 3.0$. On the other hand, there exist numerical data which suggest the absence of such a transition [14]. It has been also argued quite recently that the model in question can be mapped to the nonlinear $\sigma$ model for the unitary ensemble in which case all states are known to be localized [18]. The conclusions of papers [11,19] concerning this problem are still controversial. It is then probably fair to say that the question of whether the Anderson transition is realized in two-dimensional systems with random magnetic fluxes remains unsettled and it is a challenging problem to 
study the transport properties of such systems.

A two-dimensional system with inhomogeneous magnetic fields has been experimentally realized using the type II superconductors 20. Theoretically, a system in random magnetic fields with zero mean arises in the theory of composite fermions that describes the fractional quantum Hall effect with half-filled Landau levels [21]. It is therefore a very interesting problem to clarify not only the existence of the Anderson transition, but also the structures of the wave functions in such a system.

In this paper we have studied dynamical aspects of the electron system in static random magnetic fields by solving the time-dependent Schrödinger equation numerically. This type of analysis, which is usually called the equation-of-motion method, has been used in the research of disordered systems [22-24]. In particular, the conventional Anderson model in one- and two-dimensions, having only a diagonal disorder, has been studied by De Raedt [24] based on the exponential product formulae. However, the forth-order formula used in ref. [24] involves comutators between operators and becuase of this it is difficult to apply it directly to the system with off-diagonal disorder such as a system in random magnetic fields. We therefore adopt here the new formula for solving the time-dependent Schrödinger equation proposed by Suzuki [25. This formula has an advantage over the previous one 24, because commutators between operators are not involved. It is important to note that owing to this fact the new formula is easily applicable to a much wider class of systems including not only the systems with diagonal disorder but also those with off-diagonal disorder 26.

The quantities we have observed are the second moment of the wave packet $\left\langle r^{2}(t)\right\rangle_{c}$ defined by

$$
\left\langle r^{2}(t)\right\rangle_{c} \equiv\left\langle r^{2}(t)\right\rangle-\langle r(t)\rangle^{2}
$$


with

$$
\left\langle r^{n}(t)\right\rangle \equiv \int \mathrm{d} \Omega r^{d-1} \mathrm{~d} r r^{n}|\psi(\boldsymbol{r}, t)|^{2}
$$

where $\psi(\boldsymbol{r}, t)$ denotes the wave function at time $t$, and the temporal auto-correlation function $C(t)$ defined by

$$
\begin{aligned}
C(t) & =\frac{1}{t} \int_{0}^{t} P\left(t^{\prime}\right) \mathrm{d} t^{\prime} \\
P(t) & \equiv|\langle\psi(t) \mid \psi(0)\rangle|^{2} .
\end{aligned}
$$

Here $P(t)$ denotes the overlap function between the initial state $|\psi(0)\rangle$ and the state $|\psi(t)\rangle$ at time $t$ and $C(t)$ is its time average. These quantities have been calculated using exact eigenvalues and eigenfunctions of the system in the cases of the Harper model 27] and the critical state of the quantum Hall system 28. It has been shown that from the asymptotic behavior of the auto-correlation function $C(t)$ we can obtain information about the fractal character of the energy levels and wave functions. We thus discuss in the present work the fractal character of the wave function, which is usually observed just at the critical point or at length-scales much smaller than the characteristic length of the system. Analyses of the fractal character of the wave functions at the critical energy of the Anderson transition have been made for twodimensional systems in a strong magnetic field [29-32] and also for three-dimensional systems 33 36]. In particular, the relationship between transport properties and the fractal structures of the system is a very interesting problem [37.

The organization of the present paper is as follows. In the following section, we explain our method which is based on the higher-order decomposition of exponential operators. The expected asymptotic behaviors of the second moment of the wave packet and the auto-correlation function are also discussed in this section. In section 3, the above method is applied to the two-dimensional systems in static random 
magnetic fields and the numerical results for the growth of the second moment and the decay of the auto-correlation are presented. The final section is devoted to discussions of the physical implication of our numerical results.

\section{§2. Numerical Method}

In order to solve numerically the time-dependent Schrödinger equations, we have adopted the method based on the higher-order decomposition of exponential operators 25]. As is seen below, the method is quite general and easily applicable to complicated systems described by the tight-binding Hamiltonian with inhomogeneous hopping elements. The basic formula we have used is the fourth-order decomposition of exponential operators:

$$
\exp \left[x\left(A_{1}+A_{2}+\cdots+A_{q}\right)\right]=S(x p) S(x(1-2 p)) S(x p)+O\left(x^{5}\right)
$$

where

$$
S(x) \equiv \mathrm{e}^{x A_{1} / 2} \mathrm{e}^{x A_{2} / 2} \cdots \mathrm{e}^{x A_{q-1} / 2} \mathrm{e}^{x A_{q}} \mathrm{e}^{x A_{q-1} / 2} \cdots \mathrm{e}^{x A_{1} / 2}
$$

and the parameter $p$ is given by $p=(2-\sqrt[3]{2})^{-1}$. Here $A_{1}, \ldots, A_{q}$ are arbitrary operators.

Let us consider, in general, the tight-binding Hamiltonian on the two-dimensional square lattice that has nearest neighbor hopping amplitudes $\left\{t_{i, j} ; t_{i, j}^{*}=t_{j, i}\right\}$ and the diagonal elements $\left\{\varepsilon_{i}\right\}$ :

$$
H=-\sum_{<i, j>} t_{i, j} C_{i}^{\dagger} C_{j}+\sum_{i} \varepsilon_{i} C_{i}^{\dagger} C_{i}
$$

where $C_{i}^{\dagger}\left(C_{i}\right)$ denotes a creation (annihilation) operator of an electron at the site $i$. We then decompose this Hamiltonian into five parts (see figure 1), namely,

$$
H=\sum_{n=1}^{5} H_{n}
$$




$$
\begin{aligned}
H_{1} & \equiv \sum_{r_{x} \in \text { odd }} \sum_{r_{y}} t_{\vec{r}+\hat{x}, \vec{r}} C_{\vec{r}+\hat{x}}^{\dagger} C_{\vec{r}}+\text { h.c. } \\
H_{2} & \equiv \sum_{r_{x} \in \text { even }} \sum_{r_{y}} t_{\vec{r}+\hat{x}, \vec{r}} C_{\vec{r}+\hat{x}}^{\dagger} C_{\vec{r}}+\text { h.c. } \\
H_{3} & \equiv \sum_{r_{x}} \sum_{r_{y} \in \text { odd }} t_{\vec{r}+\hat{y}, \vec{r}} C_{\vec{r}+\hat{y}}^{\dagger} C_{\vec{r}}+\text { h.c. } \\
H_{4} & \equiv \sum_{r_{x}} \sum_{r_{y} \in \text { even }} t_{\vec{r}+\hat{y}, \vec{r}} C_{\vec{r}+\hat{y}}^{\dagger} C_{\vec{r}}+\text { h.c. } \\
H_{5} & \equiv \sum_{\vec{r}} \varepsilon_{\vec{r}} C_{\vec{r}}^{\dagger} C_{\vec{r} .}
\end{aligned}
$$

In equations (8), $\hat{x}(\hat{y})$ denotes the unit vector in the $x(y)$-direction and $r_{x}\left(r_{y}\right) \in \boldsymbol{Z}$ the $x(y)$ component of the position vector $\vec{r}$ of the sites. All the length-scales are measured in units of the lattice constant $a_{0}$. The Hamiltonian $H_{1}$, for instance, describes the hoppings between the sites whose positions are $\vec{r}$ and $\vec{r}+\hat{x}$ for $r_{x}=$ $1,3,5, \ldots$ and $r_{y}=1,2,3, \ldots$. Note that each Hamiltonian $H_{n}$ consists of operators which commute with each other, and hence we can obtain the analytical expressions for $\exp \left(-\mathrm{i} H_{n} t / \hbar\right)$ by diagonalizing two by two matrices. According to the formula (4), it is easy to see that the state vector $|\psi(t+\delta t)\rangle$ at time $t+\delta t$ can be obtained as

$$
\begin{aligned}
|\psi(t+\delta t)\rangle= & \exp (-\mathrm{i} \delta t H / \hbar)|\psi(t)\rangle \\
= & S_{2}(-\mathrm{i} \delta t p / \hbar) S_{2}(-\mathrm{i} \delta t(1-2 p) / \hbar) S_{2}(-\mathrm{i} \delta t p / \hbar)|\psi(t)\rangle \\
& +O\left(\delta t^{5}\right)
\end{aligned}
$$

with

$$
S_{2}(x) \equiv \mathrm{e}^{x H_{1} / 2} \mathrm{e}^{x H_{2} / 2} \cdots \mathrm{e}^{x H_{4} / 2} \mathrm{e}^{x H_{5}} \mathrm{e}^{x H_{4} / 2} \cdots \mathrm{e}^{x H_{2} / 2} \mathrm{e}^{x H_{1} / 2} .
$$

It should be noted that in the present approach the unitarity of the process is obviously preserved and that we have high enough accuracy to perform large time-scale simulations. It is important to note that in this method we do not need to diagonalize the whole Hamiltonian. We can thus simulate larger systems, for example a 
499 by 499 square lattice, with high precision, which is crucial if we are to extract meaningful properties from the numerical data for random systems.

In order to consider the time evolution of the wave packet with fixed energy $E$, we have carried out a numerical diagonalization of a subsystem $H_{N_{0}}$ whose size is $N_{0}$ by $N_{0}$, located at the center of the whole system and have chosen an eigenstate of $H_{N_{0}}$ with eigenvalue $E_{N_{0}} \approx E$ as the initial wave function.

Next, let us consider the asymptotic behaviors of the auto-correlation function $C(t)$ and the second moment of the wave packet $\left\langle r^{2}\right\rangle_{c}$ in several regions, namely, the metallic, the localized (insulating) and the critical regions. First, in the localized region it is clear that the second moment remains finite $\left\langle r^{2}(t)\right\rangle_{c} \rightarrow d(d+1) \xi^{2} / 4$ in the limit as $t \rightarrow \infty$, where $\xi$ denotes the localization length [24] that describes the exponential-decay of the wave function as $|\psi(r)| \propto \exp [-r / \xi]$. Obviously, the quantity $C(t)$ also goes to a nonzero value in the same limit. In contrast, in the metallic region it is expected that the second moment grows in proportion to time $t$ :

$$
\left\langle r^{2}\right\rangle_{c}=2 d D t
$$

and that the auto-correlation $C(t)$ decreases as

$$
C(t) \propto t^{-d / 2} .
$$

Here, the coefficient $D$ denotes the diffusion coefficient and $d$ the dimensionality of the system.

In the critical region where the wave function has a fractal structure, a nonconventional behavior of the auto-correlation function $C(t)$ has been observed [27, 28]. In such a case, the auto-correlation function decays in proportion to $t^{-\alpha}$ for $t \gg 1$ with $\alpha \neq d / 2$ reflecting the fractal character of the energy spectrum [27] and of 
the wave function 28]. It has been shown that the exponent $\alpha$ is related to the generalized dimension $\tilde{D}_{2}$ of the energy spectrum by $\alpha=\tilde{D}_{2}$ [27, and, further, that in the case of the quantum Hall system it is also related to the generalized fractal dimension $D_{2}$ [31] of the wave function by $\alpha=D_{2} / 2$ 28. The generalized dimension $\tilde{D}_{2}$ of the energy spectrum is defined as the scaling exponent for the correlation of energy levels:

$$
\gamma(l) \equiv \int \mathrm{d} \mu_{\psi(0)}(\omega) \int_{\omega-l}^{\omega+l} \mathrm{~d} \mu_{\psi(0)}\left(\omega^{\prime}\right) \propto l^{\tilde{D}_{2}} \quad \text { as } \quad l \rightarrow 0
$$

where $\mu_{\psi(0)}(\omega)$ denotes the spectral measure with respect to an initial state $\psi(0)$ 27]. The generalized fractal dimension $D_{2}$ of the wave function is, on the other hand, defined by:

$$
P_{2}(\lambda, E) \equiv \sum_{i}\left(\sum_{r \in \Omega_{i}(\lambda)}|\psi(r, E)|^{2}\right)^{2} \propto \lambda^{D_{2}} \quad \text { as } \quad \lambda \rightarrow 0
$$

where $\left\{\Omega_{i}(\lambda)\right\}$ denote $\lambda N \times \lambda N$ boxes that cover the whole system $(N \times N)$ 28. On the basis of these relations, the non-conventional behavior of the auto-correlation function $C(t)$ can be considered as evidence for fractal structure in the system. Since the multifractal behavior has been demonstrated not only at the critical point, but also for length-scales smaller than the characteristic length, such as the localization length in the localized region [35], a similar type of non-conventional behavior of $C(t)$ is expected if we consider length-scales much smaller than the characteristic length of the system. 


\section{§3. Model and Numerical Results}

The two-dimensional system with random magnetic fluxes is described by the tight-binding Hamiltonian:

$$
H=-\sum_{<i, j>} V_{i, j} C_{i}^{\dagger} C_{j}
$$

where

$$
V_{i, j}=V \exp \left[\mathrm{i} \theta_{i, j}\right]
$$

The phases $\left\{\theta_{i, j}\right\}$ are related to the magnetic fluxes $\left\{\phi_{i}\right\}$ through the relation:

$$
\theta_{i+\hat{x}, i}+\theta_{i+\hat{x}+\hat{y}, i+\hat{x}}+\theta_{i+\hat{y}, i+\hat{x}+\hat{y}}+\theta_{i, i+\hat{y}}=-2 \pi \phi_{i} / \phi_{0}
$$

where $\phi_{i}$ and $\phi_{0} \equiv h c /|e|$ denote the magnetic flux through the plaquette $(i, i+$ $\hat{x}, i+\hat{x}+\hat{y}, i+\hat{y}$ ) (figure 2) and the unit flux, respectively. It should be noted here that we have the gauge degrees of freedom in determining the phases $\left\{\theta_{i, j}\right\}$ for given fluxes $\left\{\phi_{i}\right\}$. The magnetic fluxes through each plaquettes of the square lattice are distributed uniformly in the interval $\left[-\phi_{0} / 2, \phi_{0} / 2\right]$, and the fluxes are assumed to have no spatial correlation, i.e.

$$
\left\langle\phi_{i} \phi_{j}\right\rangle_{\mathrm{av}}=\frac{1}{12} \phi_{0}^{2} \delta_{i j}
$$

Here the angular bracket \langle\rangle$_{\text {av }}$ denotes the average over the distribution of fluxes.

We have calculated the auto-correlation function $C(t)$ and the second moment of the wave packet $\left\langle r^{2}\right\rangle_{c}$ at various energies. The sizes of the systems are 499 by 499 for the energies near the band center and 199 by 199 for the energy near the band edge. We have carried out an exact diagonalization for the 21 by 21 subsystem at the center of the system and taken the eigenfunction of the subsystem whose eigenvalue is closest to the given energy $E$ as the initial wave packet. By this procedure we can 
simulate the diffusion of the wave packet whose energy is approximately equal to $E$. The single time step $\delta t$ is taken to be $0.2(\hbar / V)$ in the simulation. With this choice of $\delta t$, fluctuations of the expectation value of the Hamiltonian

$$
\langle H\rangle \equiv\langle\psi(t)|H| \psi(t)\rangle
$$

can be safely neglected [24 throughout our simulations $(t \leq 8000(\hbar / V))$. In the actual simulation, the quantities $\left\langle r^{2}\right\rangle_{c}$ and $\log C(t)$ are averaged over five realizations of random magnetic flux distribution.

Let us first discuss the diffusion near the band edge. The states near the band edge are expected to be localized [11, 14]. Our results for the energy $\langle H\rangle \sim-3.35 \mathrm{~V}$ are shown in figures 3(a) and 3(b), which is based on a calculation of five configurations of random magnetic fields. The second moment of the wave packet $\left\langle r^{2}\right\rangle_{c}$ seems to have a finite value in the limit $t \rightarrow \infty$. The localization length can be roughly estimated as $\xi \sim 14$, which is consistent with the value obtained from the finite-size scaling analysis of ref. [14]. It may not be clear from the behavior of the second moment of the wave packet alone whether the states are indeed localized or not. The behavior of the auto-correlation $C(t)$, however, clearly suggests that the state at time $t$ has a nonzero overlap integral with the initial state in the limit $t \rightarrow \infty$ and thus the states are localized in this energy region.

Next, we discuss the diffusion near the band center. The results for the energies $\langle H\rangle / V \sim 0,-1,-2,-2.6$ are presented in figures $4,5,6$ and 7 , respectively. The growth of the second moment of the wave packets turns out to be diffusive; the numerical value of the exponent $a$ for the power-law growth defined in

$$
\left\langle r^{2}(t)\right\rangle_{c} \propto t^{a}
$$

is very close to 1 . It should be noted here that, in the critical case of the Harper 
model, the exponent $a$ has been estimated to be about 0.97 [38, 39], which is also close to 1 40. The exponent $a$ has been also estimated to be 1 for the critical states of the quantum Hall system [28]. By assuming a linear dependence of $\left\langle r^{2}(t)\right\rangle$ with respect to $t$, we can evaluate the diffusion coefficient $D$ defined in eq.(11), which is related to the conductance of the system. The obtained values of the diffusion coefficients for energies $-2.6,-2,-1$ and 0 are consistent with those obtained using the Landauer formula 44].

On the other hand, the exponents $\alpha$ for the power-law decay of the auto-correlation function show deviations from the value $d / 2=1$ in the same energy region. For the energy region $-2.6 \leq E \leq 0$, the exponent $\alpha$ is estimated to vary from 0.9 to 0.88 , which is smaller than 1 . These deviations suggest the presence of fractal properties of the energy spectrum and the wave function, just as observed in the cases of the Harper model and the quantum Hall system [27, 28]. The deviations themselves are smaller than that obtained for the critical case of the quantum Hall system [28], suggesting a larger fractal dimensionality of the present system.

\section{§4. Discussions}

We have performed numerical simulations for the diffusion of electrons in static random magnetic fields using a new formula for the decomposition of exponential operators 25]. The systems we have treated are much larger than those in previous studies of this kind [24, 28]. The present method reproduces the results previously found from the finite-size scaling analyses, namely that the states near the band edge are localized. On the other hand, away from the band edge, the second moment of the wave packets increases linearly in time, which suggests that the conductance is non-zero in this region. If the system is described by the conventional diffusion 
equation, the exponent $\alpha$ should be $d / 2=1$ and in that case no fractal structure is present. However, the obtained values of the exponents $\alpha$ of the power-law decay of the auto-correlation function are smaller than $d / 2=1$. It is expected from these deviations of the values of $\alpha$ from 1 that some kind of fractal structure exists in the energy spectrum as well as in the eigenfunctions of the present system. This type of behavior, namely the linear increase of $\left\langle r^{2}\right\rangle_{c}$ in time and the non-conventional exponents for the power-law decay of the auto-correlation function, has been observed in the critical case of the Harper model and the critical states in the quantum Hall system [27,28]. It may therefore be natural to suppose that the states away from the band edge in the present system with random magnetic fluxes are also critical.

It has been shown that, by assuming conformal invariance, the exponent $\eta$ for the power-law decay of the critical wave function in two-dimensional systems can be related to the exponential decay-length of the Green function in quasi-one dimensional strip systems 41, 42]. The exponent $\eta$, which describes the power-law decay of the density correlation by $\left\langle|\psi(r)|^{2}|\psi(0)|^{2}\right\rangle_{\text {av }} \sim|r|^{-2 \eta}$ in two dimensions, is expressed as 42

$$
\eta=\frac{1}{\pi \Lambda_{c}}
$$

where $\Lambda_{c}$ denotes the renormalized localization length, defined by

$$
\Lambda_{c} \equiv \lim _{M \rightarrow \infty} \xi_{M}(E) / M
$$

Here $\xi_{M}(E)$ denotes the localization length along a strip of width $M$ with energy $E$ corresponding to the critical states in infinite two-dimensional systems [43]. The fractal dimensionality $d^{*}$ of the wave function is then obtained from the exponent $\eta$ as 42

$$
d^{*}=d-2 \eta
$$


In this way, the fractal dimensionality $d^{*}$ of the wave function in a two-dimensional system at its critical energy, or at the length-scales much smaller than the characteristic length, can thus be evaluated from the scaling behavior of the localization length along the quasi-one-dimensional systems. Making use of this theory [42], we have performed independently the calculation of $\xi_{M}$ for a few random field configurations in strips whose sizes are $M \times L$ with $L=10^{5}$ and $M=8,12,16,20,34,40$ and 50 in units of the lattice spacing $a_{0}$ (figure 8) and have estimated the exponent $\eta$ through the formulae (21) and (22). The values of $d^{*}$ obtained by the formula (23) are then compared with the generalized fractal dimension $D_{2}$ obtained from the exponent $\alpha$ by assuming the relation $D_{2}=2 \alpha$ [28] (Table 1). It is rather remarkable that the values of $d^{*}$ and $D_{2}$ coincide fairly well with each other.

We have thus argued, based on our numerical results, that there is evidence for fractal structure in the energy spectrum and the eigenfunction in the region away from the band edge. However, we admit that the fractal character of the present system turns out to be not so strong compared with the case of the critical states in the quantum Hall system [28]. At the band center, in particular, we cannot exclude the possibility that there is no fractal structure, because the value of the exponent $\alpha$ turns out to be rather close to 1 . Since the fractal structure is observed when the characteristic length is much larger than the size of the system, then precisely speaking, our results do not distinguish the critical states from the localized states with extremely large localization length. Simulations in much larger systems and the investigation of the probability distributions of $\left\langle r^{2}\right\rangle_{c}$ and $C(t)$ will be needed in order to obtain more detailed information about the thermodynamic properties of this system.

Finally, we emphasize that the formula we have used in this work is quite general. 
It would be very interesting to study many other dynamical problems, such as the anomalous diffusion [45] and the mesoscopic dynamical echo [46] by making use of the present method.

\section{Acknowledgments}

The authors would like to thank Profs. Y. Ono, J. Kondo, B. Kramer, M. Kohmoto and M. Takahashi for fruitful discussions. They also thank Dr. S.M. Manning for reading the manuscript. Numerical calculations were performed on HITAC S-3800 of the Computer Center, University of Tokyo. This work was partly supported by the Grant-in-Aid No. 06740316, No. 04231105 and No. 05854024, from the Ministry of Education, Science and Culture, Japan. One of the authors (T. K.) thanks the Fūjukai Foundation for a scholarship.

\section{References}

[1] E. Abrahams, P.W. Anderson, D.C. Licciardello and T.V. Ramakrishnan, Phys. Rev. Lett. 42, 673 (1979).

[2] A. Kawabata, Prog. Theor. Phys. Suppl. 84, 16 (1985).

[3] P.A. Lee and T.V. Ramakrishnan, Rev. Mod. Phys. 57, 287 (1985).

[4] "Anderson Transition and Mesoscopic Fluctuations" (edited by B. Kramer and G. Schön), Physica A167,pp 1-314 (1990).

[5] Edited by B. Kramer, "Quantum Coherence in Mesoscopic Systems", Plenum Press (1991).

[6] B. Kramer and A. MacKinnon, Rep. Prog. Phys. 56, 1469 (1993). 
[7] B.L. Altshuler and L.B. Ioffe, Phys. Rev. Lett. 692979 (1992).

[8] A. Barelli, R. Fleckinger and T. Ziman, Phys. Rev. B49, 3340 (1994).

[9] G. Gavazzi, J.M. Wheatley and A.J. Schofield, Phys. Rev. B47, 15170 (1993).

[10] T. Ohtsuki, Y. Ono and B. Kramer, J. Phys. Soc. Jpn. 63, 685 (1994).

[11] C. Pryor and A. Zee, Phys. Pev. B46, 3116 (1992).

[12] V. Kalmeyer and S.C. Zhang, Phys. Rev. B46, 9889 (1992).

[13] Y. Avishai, Y. Hatsugai and M. Kohmoto, Phys. Rev. B47, 9561 (1993).

[14] T. Sugiyama and N. Nagaosa, Phys. Rev. Lett. 70, 1980 (1993).

[15] V. Kalmeyer, D. Wei, D.P. Arovas and S.C. Zhang, Phys. Rev. B48, 11095 (1993).

[16] S.C. Zhang and D.P. Arovas, Phys. Rev. Lett. 72, 1886 (1994).

[17] D.K.K. Lee and J.T. Chalker, Phys. Rev. Lett. 72, 1510 (1994).

[18] A.G. Aronov, A.D. Mirlin and P. Wölfle, Phys. Rev. B49, 16609 (1994).

[19] D.Z. Liu, X.C. Xie, S. Das Sarma and S.C. Zhang, unpublished.

[20] A.K. Geim, S.J. Bending, I.V. Grigorieva and M.G. Blamire, Phys. Rev. B49, 5749 (1994).

[21] B.I. Halperin, P.A. Lee and N, Read, Phys. Rev. B47, 7312 (1993).

[22] D. Weaire and A.R. Williams, J. Phys. C10, 1239 (1977).

[23] B. Kramer, A. MacKinnon and D. Weaire, Phys. Rev. B23, 6357 (1981). 
[24] H. De Raedt, Computer Phys. Rep. 7, 1 (1987); Europhys. Lett. 3, 139 (1987).

[25] M. Suzuki, Phys. Lett. A146, 319 (1990); J. Math. Phys. 32, 400 (1991); Phys. Lett. A165, 387 (1992); J. Phys. Soc. Jpn. 61, 3015 (1992); Commun. Math. Phys. 163, 491 (1994).

[26] H. De Raedt and K. Michielsen, Computers in Phys. 8, 600 (1994).

[27] R. Ketzmerick, G. Petschel and T. Geisel, Phys. Rev. Lett. 69, 695 (1992).

[28] B. Huckestein and L. Schweitzer, Phys. Rev. Lett. 72, 713 (1994).

[29] H. Aoki, J. Phys. C: Solid State Phys. 16, L205 (1983) ; Phys. Rev. B33, 7310 (1986).

[30] Y. Ono, T. Ohtsuki and B. Kramer, J. Phys. Soc. Jpn. 60, 270 (1991).

[31] W. Pook and M. Janßen, Z. Phys. B82, 295 (1991).

[32] B. Huckestein, B. Kramer and L. Schweitzer, Surface Science 263, 125 (1992).

[33] S.N. Evangelou, J. Phys. A: Math. Gen. 23, L317 (1990).

[34] T.M. Chang, J.D. Bauer and J.L. Skinner, J. Chem. Phys. 938973 (1990).

[35] M. Schreiber and H. Grussbach, Phys. Rev. Lett. 67, 607 (1991); Mod. Phys. Lett. B6, 851 (1992).

[36] H. Grussbach and M. Schreiber, Physica A191, 394 (1992).

[37] T. Brandes, L. Schweitzer and B. Kramer, Phys. Rev. Lett. 72, 3582 (1994).

[38] H. Hiramoto and S. Abe, J. Phys. Soc. Jpn. 57, 1365 (1988). 
[39] H. Hiramoto and M. Kohmoto, Int. J. Mod. Phys. B6, 281 (1992).

[40] M. Wilkinson and E.J. Austin, Phys. Rev. B50, 1420 (1994).

[41] J.L. Cardy, J. Phys. A: Math. Gen. 17, L385 (1984).

[42] J.L.Pichard and G. Sarma, J. Phys. C: Solid State Phys. 18, 3457 (1985).

[43] A. MacKinnon and B. Kramer, Phys. Rev. Lett. 47, 1546 (1981); Z. Phys. B53, 1 (1983).

[44] T. Ohtsuki, K. Slevin and Y. Ono, J. Phys. Soc. Jpn. 62, 3979 (1993).

[45] P. Sebbah, D. Sornette and C. Vanneste, Phys. Rev. B48, 12506 (1993).

[46] V.N. Prigodin, B.L. Altshuler, K.B. Efetov and S. Iida, Phys. Rev. Lett. 72, 546 (1994). 


\begin{tabular}{cccc}
\hline$E$ & $\Lambda_{c}$ & $d^{*}=d-2 \eta$ & $D_{2}=2 \alpha$ \\
\hline \hline 0 & $3.04 \pm 0.04$ & $1.791 \pm 0.002$ & $1.8 \pm 0.06$ \\
\hline-1 & $3.00 \pm 0.08$ & $1.788 \pm 0.006$ & $1.8 \pm 0.06$ \\
\hline-2 & $2.73 \pm 0.07$ & $1.766 \pm 0.006$ & $1.8 \pm 0.03$ \\
\hline-2.6 & $2.34 \pm 0.09$ & $1.73 \pm 0.01$ & $1.76 \pm 0.02$ \\
\hline
\end{tabular}

Table 1: Fractal dimensions obtained from the exponents $\eta$ and those from the exponents $\alpha$ for $E=0,-1,-2,-2.6$. The values $\Lambda_{c}$ are estimated using $M \times L$ strips with $8 \leq M \leq 50$ and $L=10^{5}$. 


\section{Figure Captions}

Fig. 1: The decomposition of the Hamiltonian. The thin solid, thick solid, thin dashed and thick dashed lines correspond to the Hamiltonian $H_{1}, H_{2}, H_{3}$ and $H_{4}$ in equation (8), respectively.

Fig. 2: The plaquette $(i, i+\hat{x}, i+\hat{x}+\hat{y}, i+\hat{y})$ and the flux $\phi_{i}$ through it.

Fig. 3: (a) The growth of the second moment $\left\langle r^{2}(t)\right\rangle_{c}$ of the wave packet and (b) the time-dependence of the auto-correlation function $C(t)$ for the energy $\langle H\rangle \simeq-3.35(-3.35 \pm 0.01) V$. The bars around the data points in figures $3,4,5,6$ and 7 indicate the fluctuations of the mean values with respect to five realizations of random magnetic field configurations.

Fig. 4: (a) The growth of the second moment $\left\langle r^{2}(t)\right\rangle_{c}$ of the wave packet and (b) the time-dependence of the auto-correlation function $C(t)$ for the energy $\langle H\rangle \simeq 0\left( \pm 1.0 \times 10^{-4}\right) V$. The solid line in (a) corresponds to $D=1.59 a_{0}^{2} V / \hbar$. The exponent $\alpha$ is estimated to be $0.90 \pm 0.03$. For comparison, the conventional decay of $C(t) \propto t^{-1}$ is also shown by the dashed lines in figures 4(b), 5(b), 6(b) and 7(b).

Fig. 5: (a) The growth of the second moment $\left\langle r^{2}(t)\right\rangle_{c}$ of the wave packet and (b) the time-dependence of the auto-correlation function $C(t)$ for the energy $\langle H\rangle \simeq-1(-1.01 \pm 0.02) \mathrm{V}$. The solid line in (a) corresponds to $D=$ $1.54 a_{0}^{2} V / \hbar$. The exponent $\alpha$ is estimated to be $0.90 \pm 0.03$.

Fig. 6: (a) The growth of the second moment $\left\langle r^{2}(t)\right\rangle_{c}$ of the wave packet and (b) the time-dependence of the auto-correlation function $C(t)$ for the energy 
$\langle H\rangle \simeq-2(-1.99 \pm 0.01) V$. The solid line in (a) corresponds to $D=$ $1.33 a_{0}^{2} V / \hbar$. The exponent $\alpha$ is estimated to be $0.90 \pm 0.014$.

Fig. 7: (a) The growth of the second moment $\left\langle r^{2}(t)\right\rangle_{c}$ of the wave packet and (b) the time-dependence of the auto-correlation function $C(t)$ for the energy $\langle H\rangle \simeq-2.6(-2.61 \pm 0.01) V$. The solid line in (a) corresponds to $D=$ $0.98 a_{0}^{2} V / \hbar$. The exponent $\alpha$ is estimated to be $0.88 \pm 0.01$.

Fig. 8: Energy dependence of the exponential decay length $\xi_{M}$ divided by the width of the strip $M$ [14, 43]. The circle, square, diamond, triangle, bullet, painted square and painted diamond correspond to $M=8.12,16,20,30,40$ and 50 respectively. The length of the strip $L$ is $10^{5}$ and the average is performed over more than two random flux configurations. Due to the exact particle-hole symmetry, only the negative energy region is plotted. 